\title{
Characterization of transition to chaos with multiple positive Lyapunov exponents by unstable periodic orbits
}

\author{
Ruslan Davidchack ${ }^{\mathrm{a}}$, Ying-Cheng Lai ${ }^{\mathrm{b}, *}$ \\ a Department of Physics and Astronomy, University of Kansas, Lawrence, KS 66045, USA \\ ${ }^{\mathrm{b}}$ Departments of Mathematics and Electrical Engineering, Center for Systems Science and Engineering Research, Arizona State University, \\ Tempe, AZ 85287, USA
}

Received 2 February 2000; received in revised form 28 April 2000; accepted 3 May 2000

Communicated by C.R. Doering

\begin{abstract}
We investigate how the transition to chaos with multiple positive Lyapunov exponents can be characterized by the set of infinite number of unstable periodic orbits embedded in the chaotic invariant set. We argue and provide numerical confirmation that the transition is generally accompanied by a nonhyperbolic behavior: unstable dimension variability. As a consequence, the Lyapunov exponents, except for the largest one, pass through zero continuously. (C) 2000 Elsevier Science B.V. All rights reserved.
\end{abstract}

PACS: 05.45.Jn; 05.45.Ac

A fundamental question in nonlinear dynamics is to understand how chaos arises and evolves as a system parameter changes. It is now known that there are four possible routes to low-dimensional chaos, i.e., chaos with only one positive Lyapunov exponent: $(i)$ the period-doubling cascade route [1]; (ii) the intermittency transition route [2]; (iii) the crisis route [3]; and (iv) the route to chaos in quasiperiodically driven systems [4-6]. Transition to chaos with multiple positive Lyapunov exponents has become a recent topic of study (see, e.g., Refs. [7-12]). A good understanding of this transition is of interest because high-dimensional phenomena are re-

\footnotetext{
* Corresponding author. Fax: +1-480-965-8119.

E-mail address: yclai@chaos1.la.asu.edu (Y.-C. Lai).
}

ceiving an ever increasing attention (see, e.g., Refs. $[13,14])$, and there have been extensive efforts in the control (see, e.g., Refs. [15-19]) and synchronization (see, e.g., Refs. [20-22]) of high-dimensional chaotic systems.

In order to better understand a transition, it is desirable to study it in terms of the fundamental dynamical quantities of the system. There is nothing more fundamental than to characterize a transition by unstable periodic orbits embedded in the dynamical invariant set $^{1}$. Most known bifurcations in low-di-

\footnotetext{
${ }^{1}$ It has been a widely accepted notion that in deterministic chaotic systems, unstable periodic orbits constitute the skeleton of the chaotic sets such as chaotic attractors or nonattracting chaotic saddles. See, for example, [23].
} 
mensional chaos theory, such as the period-doubling bifurcation [1], the saddle-node bifurcation, crises [3], etc., are characterized by properties of some key unstable periodic orbits involved in the bifurcations. The aim of this paper is to present a periodic-orbit characterization of the transition to chaos with multiple positive Lyapunov exponents. Our principal result is that the transition is generally accompanied by severe unstable dimension variability [24], a type of nonhyperbolicity that has been documented recently for high-dimensional chaotic systems and is believed to be common in such systems [25-27]. Specifically, assuming that the system already possesses one positive Lyapunov exponent, we find that unstable dimension variability provides a possible dynamical mechanism for any subsequent Lyapunov exponents to become positive as a system parameter changes. As a consequence, the transition is typically smooth in the sense that the subsequent Lyapunov exponents pass through zero continuously. We note that the smooth behavior of Lyapunov exponents in high-dimensional chaotic systems has been observed previously [7-12], but our results here provide an explanation for this behavior based on unstable periodic orbits. In what follows, we will first present a heuristic theory for the transition and provide explicit numerical confirmation. A key factor that enables us to perform numerical test of our idea is the availability of an algorithm for computing a large number periodic orbits for general dynamical systems [28,29], which we have implemented with substantial improvements [30].

Consider a dynamical system described by the following $N$-dimensional map: $\boldsymbol{x}_{n+1}=\boldsymbol{F}\left(\boldsymbol{x}_{n}, a\right)$, where $\boldsymbol{x} \in \mathscr{R}^{N}(N>2)$ and $a$ is a bifurcation parameter. Since we are interested in the transition to chaos with more than one positive Lyapunov exponent, we assume that near the transition, the system has a chaotic attractor with at least one positive Lyapunov exponent. For concreteness, we let the transition point be $a_{\mathrm{c}}$ and assume that for $a \leqq a_{\mathrm{c}}$, there is one positive Lyapunov exponent $\left(\lambda_{1}>0\right)$ and for $a \gtrsim a_{\mathrm{c}}$, there are two positive ones $\left(\lambda_{1}>\lambda_{2}>0\right)$. Thus, near $a_{\mathrm{c}}$, each periodic orbit embedded in the attractor has at least one expanding direction. Consider periodic orbits of period $p$. There are $N_{p} \sim \mathrm{e}^{h_{\mathrm{T}} p}$ of them, where $h_{\mathrm{T}}>0$ is the topological entropy of the attractor. Since $\lambda_{1}$ is positive and the parameter interval of interest is small (near $a_{\mathrm{c}}$ ), $h_{\mathrm{T}}$ remains approximately constant in this interval ${ }^{2}$. In order to relate the Lyapunov exponents to unstable periodic orbits, we note two facts: (1) the exponents are defined with respect to the natural measure of the chaotic attractor [34], and (2) a typical trajectory on the attractor visits the neighborhoods of the infinite number of unstable periodic orbits. Thus, although periodic orbits are atypical in the sense that they form a set of Lebesgue measure zero, the natural measure can be related to the infinite number of atypical measures associated with unstable periodic orbits. In particular, it was shown rigorously for hyperbolic systems [35] and verified numerically for more general chaotic systems [36] that the natural measure $\mu_{S}$ in a phase space region $S$ can be expressed as:

$\mu_{S}=\lim _{p \rightarrow \infty} \sum_{j \in S} \mathrm{e}^{-\lambda_{1}^{(j)} p}$,

where $\lambda_{i}^{(j)}$ 's $(i=1, \ldots, N)$ are the eigenvalues of the $j$ th periodic orbit of period $p, \lambda_{1}^{(j)}>0 \quad(j=$ $\left.1, \ldots, N_{p}\right)$, and the summation is over all periodic orbits of period $p$ and its integer factors contained in $S$. The Lyapunov exponents of the attractor can then be written, approximately, as:

$\lambda_{i} \approx \lim _{p \rightarrow \infty} \sum_{j=1}^{N_{p}} \lambda_{i}^{(j)} \mathrm{e}^{-\lambda_{1}^{(j)} p}, \quad i=1, \ldots, N$,

where the expression becomes exact if the underlying dynamics is hyperbolic [35]. We can now examine the second Lyapunov exponent:

$\lambda_{2} \approx \lim _{p \rightarrow \infty} \sum_{j=1}^{N_{p}} \lambda_{2}^{(j)} \mathrm{e}^{-\lambda_{1}^{(j)} p}$,

where $\lambda_{2} \lesssim 0$ for $a \lesssim a_{\mathrm{c}}$ and $\lambda_{2} \gtrsim 0$ for $a \gtrsim a_{\mathrm{c}}$. Since there are approximately equal numbers of un-

\footnotetext{
${ }^{2}$ For typical Hamiltonian systems and for Axiom-A attractors of dissipative dynamical systems, it was proven that $h(\mu)=$ $\sum_{\lambda_{i}>0} \lambda_{i}$, where $h(\mu)$ is the metric entropy and $\lambda_{i}>0(i=1, \ldots)$ are positive Lyapunov exponents (see Ref. [31], reprinted in Ref. [32], and Ref [33]). Generally, the topological and metric entropies satisfy $h_{\mathrm{T}} \geq h(\mu)$ [33]. In our case, because: (1) $\lambda_{1} \approx$ constant $>0$ and $\lambda_{2} \approx 0$, and (2) the derivative of the Lyapunov exponents with respect to the bifurcation parameter is bounded, we expect $h_{\mathrm{T}}$ to be approximately constant about the transition.
} 
stable periodic orbits immediately before and after the transition, and the dynamical properties of the periodic orbits are structurally stable, we see that it is generally not possible for all $\lambda_{2}^{(j)}\left(j=1, \ldots, N_{p}\right)$ to be negative for $a \leq a_{\mathrm{c}}$ and all to be positive for $a \gtrsim a_{\mathrm{c}}$. The only possibility for $\lambda_{2}$ to change sign at $a_{\mathrm{c}}$ is for some $\lambda_{2}^{(j)}$ to be negative and some to be positive for $a$ near $a_{\mathrm{c}}$, with a balance achieved at $a_{\mathrm{c}}$. Specifically, let:

$$
\begin{aligned}
& \lambda_{2}^{\mathrm{s}}(a)=\lim _{p \rightarrow \infty} \sum_{\lambda_{2}^{(j)}<0} \lambda_{2}^{(j)} \mathrm{e}^{-\lambda_{1}^{(j)} p}, \\
& \lambda_{2}^{\mathrm{u}}(a)=\lim _{p \rightarrow \infty} \sum_{\lambda_{2}^{(j)}>0} \lambda_{2}^{(j)} \mathrm{e}^{-\lambda_{1}^{(j)} p},
\end{aligned}
$$

where $\lambda_{2}^{\mathrm{s}}(a)$ and $\lambda_{2}^{\mathrm{u}}(a)$ are the stable and unstable weights associated with the second largest Lyapunov exponent. We see that the transition to chaos with multiple positive Lyapunov exponents can occur via the following scenario: (1) $\left|\lambda_{2}^{\mathrm{s}}(a)\right|>\lambda_{2}^{\mathrm{u}}(a)$ for $a \lesssim$ $a_{\mathrm{c}}$; (2) $\left|\lambda_{2}^{\mathrm{s}}(a)\right|<\lambda_{2}^{\mathrm{u}}(a)$ for $a \gtrsim a_{\mathrm{c}}$; and (3) $\left|\lambda_{2}^{\mathrm{s}}(a)\right|=$ $\lambda_{2}^{\mathrm{u}}(a)$ at $a=a_{\mathrm{c}}$. Two consequences associated with this scenario become immediately apparent: (1) near the transition, there is severe unstable dimension variability because unstable periodic orbits can have different number of unstable directions, and (2) $\lambda_{2}$ passes through zero at $a_{\mathrm{c}}$ continuously. We stress that the key element in our argument for the continuity of the transition is the continuous dependence of the largest Lyapunov exponent on $a$ in the vicinity of $a_{\mathrm{c}}$.

The above scenario for the transition to chaos with multiple positive Lyapunov exponents is based on the characterization of the natural measure by unstable periodic orbits embedded in the attractor, i.e., Eq. (1), which is rigorously valid only when the system is hyperbolic. We expect, however, Eq. (1) to hold, at least approximately, for nonhyperbolic chaotic systems as well. To give a supporting argument, we recall the formal definition of hyperbolicity. A chaotic set is hyperbolic if: $(i)$ at each point of the trajectory the phase space can be split into an expanding and a contracting subspaces and, the expanding subspace evolves into the expanding one along the trajectory and the same is true for the contracting subspace; (ii) the angle between the stable and the unstable subspaces is bounded away from zero. In low-dimensional chaotic systems with only one positive Lyapunov exponent, nonhyperbolicity occurs typically because of the violation of condition (ii), i.e., there are tangencies between the stable and the unstable subspaces. The tangencies are, however, rare in the sense that the set of tangency points has Lebesgue measure zero. In this case, Eq. (1) is expected to be valid [36]. In high-dimensional systems, nonhyperbolicity can also occur due to the violation of condition $(i)$, i.e., due to unstable dimension variability [25-27]. From Eq. (1), we see that the natural measure is determined by the largest Lyapunov exponent. Thus, in so far as there exists at least one unstable direction for every point in the chaotic set (corresponding to one positive Lyapunov exponent), we expect Eq. (1), and consequently Eqs. (2)-(4), to be valid. Since the argument assumes that the largest Lyapunov exponent remains positive, we see that it does not apply to the evolution of the largest exponent itself. Thus, the routes to chaos with one and more than one positive Lyapunov exponent are generally different.

As our representative numerical example, we consider the following four-dimensional system consisting of two coupled Ikeda-Hammel-Jones-Moloney (IHJM) maps [37,38]:

$$
\begin{aligned}
& x_{n+1}=a+b\left(x_{n} \cos \phi_{n}-y_{n} \sin \phi_{n}\right), \\
& y_{n+1}=b\left(x_{n} \sin \phi_{n}+y_{n} \cos \phi_{n}\right), \\
& x_{n+1}^{\prime}=a+b\left(x_{n}^{\prime} \cos \phi_{n}^{\prime}-y_{n}^{\prime} \sin \phi_{n}^{\prime}\right), \\
& y_{n+1}^{\prime}=b\left(x_{n}^{\prime} \sin \phi_{n}^{\prime}+y_{n}^{\prime} \cos \phi_{n}^{\prime}\right),
\end{aligned}
$$

with the couplings between $(x, y)$ and $\left(x^{\prime}, y^{\prime}\right)$ occurring in the phase variables $\phi_{n}$ and $\phi_{n}^{\prime}$ :

$$
\begin{aligned}
& \phi_{n}=k-d /\left(1+x_{n}^{2}+y_{n}^{2}\right)+2 \pi \epsilon\left(x_{n}^{\prime}-x_{n}\right), \\
& \phi_{n}^{\prime}=k-d /\left[1+\left(x_{n}^{\prime}\right)^{2}+\left(y_{n}^{\prime}\right)^{2}\right]+2 \pi \epsilon\left(x_{n}-x_{n}^{\prime}\right),
\end{aligned}
$$

where $a, b, k$, and $d$ are parameters, and $\epsilon$ represents the coupling strength. The IHJM map models 
the dynamics of an optical pulse propagating in a ring cavity, subject to partial reflection, phase and amplitude modulation and distortion due to a nonlinear optical medium in the cavity. We conceive that the phase coupling in Eq. (6) is physically meaningful as phase interaction is readily realizable in nonlinear optics. In our numerical experiments, we fix $(a, b, k, d)=(0.85,0.9,0.4,5.18)$ so that the uncoupled IHJM map exhibits a chaotic attractor with one positive Lyapunov exponent, and we choose $\epsilon$ as the bifurcation parameter. Fig. 1 shows the four Lyapunov exponents of Eq. (5) versus $\epsilon$, where we see that a transition occurs at $\epsilon=\epsilon_{\mathrm{c}} \approx 0.185$ : there is one positive Lyapunov exponent for $\epsilon<\epsilon_{\mathrm{c}}$ and there are two for $\epsilon>\epsilon_{\mathrm{c}}$. Apart from regimes of: (1) periodic windows, and (2) wild fluctuations of the exponents in which there are multiple coexisting attractors, we see that the second largest Lyapunov exponent $\lambda_{2}$ (or the envelope of $\lambda_{2}$ ) appears to pass through zero continuously as $\epsilon$ is increased through $\epsilon_{\mathrm{c}}$, as shown in the inset of Fig. 1.

We now provide evidence that severe unstable dimension variability occurs near $\epsilon_{\mathrm{c}}$. To compute unstable periodic orbits embedded in the chaotic

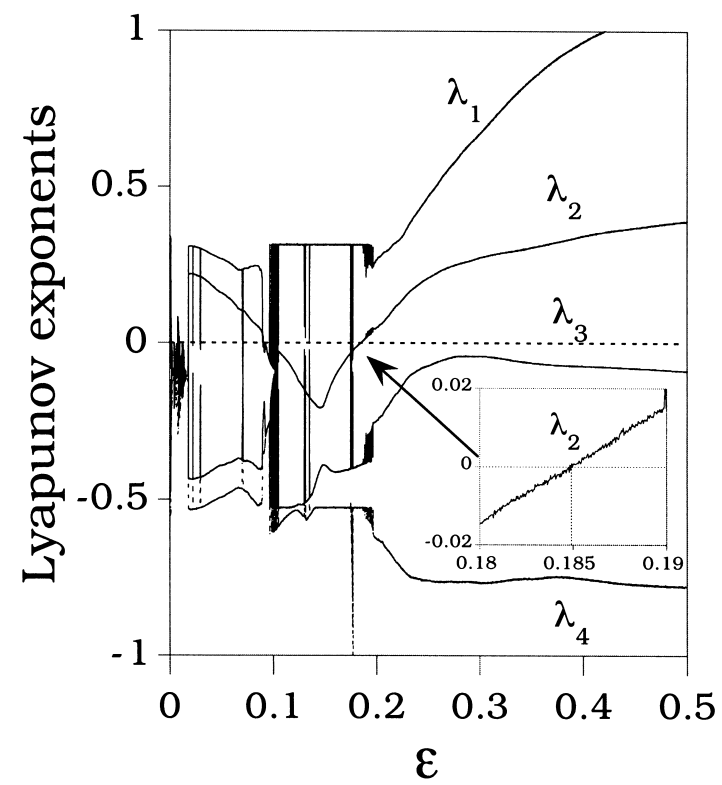

Fig. 1. The four Lyapunov exponents of Eq. (5) versus $\epsilon$. A blowup of the behavior of $\lambda_{2}$ near $\epsilon_{\mathrm{c}}$, a transition point to chaos with two positive Lyapunov exponents, is shown in the inset.
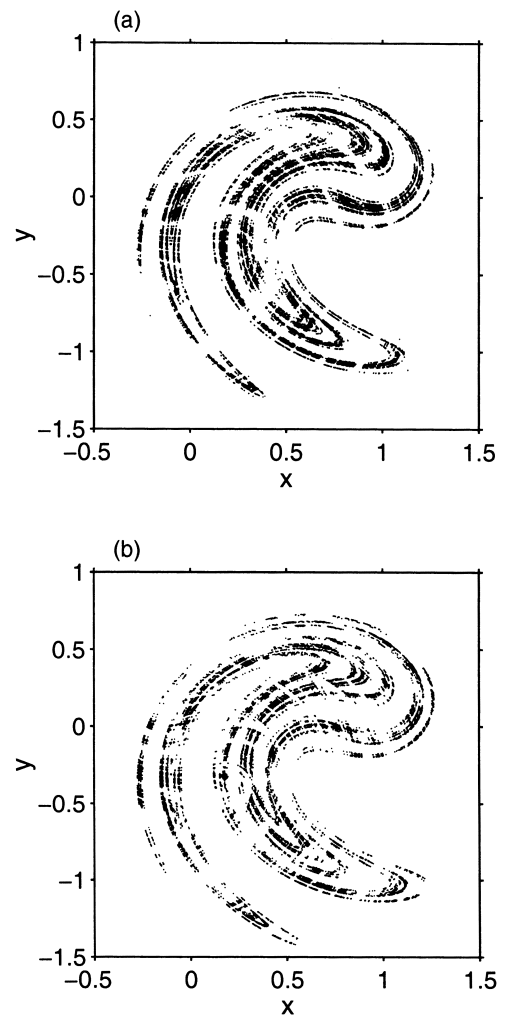

Fig. 2. Locations of unstable periodic orbits of periods 24 and 25 in the synchronization manifold $\mathscr{M}$ with unstable dimensions one and two are shown in (a) and (b), respectively.

attractor of Eq. (5), we make use of an improved version [30] of the Schmelcher-Diakonos algorithm $[28,29]$ which can, in principle, yield complete sets of periodic orbits of relatively large period ${ }^{3}$. The algorithm is particularly powerful for low-dimensional maps such as the two-dimensional IHJM map for which we find a total of over $10^{5}$ orbit points of period up to 25 . For the four-dimensional coupled system Eq. (5), due to a much larger topological entropy, we detected periodic orbits of period only up to 7 for a total of about 20000 orbit points. To make numerical experiments feasible, we note that

\footnotetext{
${ }^{3}$ Even though we cannot prove rigorously the completeness of the detected sets of orbits, our algorithm allows us to conduct a thorough exploration of the phase space, so that the number of undetected orbits, if any, is extremely small.
} 
the synchronization manifold defined by $\mathscr{M}:\{x=$ $\left.x^{\prime}, y=y^{\prime}\right\}$ is actually a solution of Eq. (5). In $\mathscr{M}$, the invariant set is the chaotic attractor of the IHJM map for which a large number of periodic orbits can be computed. These periodic orbits are a subset of all periodic orbits embedded in the chaotic attractor of Eq. (5) because $\mathscr{M}$ is part of the attractor. In situations where $\mathscr{M}$ is transversely stable, the chaotic attractor in $\mathscr{M}$ is an attractor of the full system and, hence, periodic orbits in $\mathscr{M}$ are all orbits of Eq. $(5)^{4}$ Fig. 2 shows the locations of the unstable periodic orbits of periods 24 and 25 in $\mathscr{M}$ for $\epsilon=0.19 \gtrsim \epsilon_{\mathrm{c}}$, where the transversely stable and unstable orbits are shown in (a) and (b), respectively. Unstable dimension variability is clearly present because the transversely stable orbits have unstable dimension one and the transversely unstable ones have two. In fact, unstable dimension variability is severe ${ }^{5}$ because each of the two groups of periodic orbits appears to cover densely the whole chaotic attractor in $\mathscr{M}$.

We have also computed unstable periodic orbits of the full four-dimensional system Eq. (5) within our computational resource. The total numbers of periodic orbits of these periods actually change slightly as $\epsilon$ increases through $\epsilon_{\mathrm{c}}$. Fig. 3 shows, for $\epsilon=0.185 \approx \epsilon_{\mathrm{c}}$, histograms of the weighted Lya-

\footnotetext{
${ }^{4}$ We find that near $\epsilon_{\mathrm{c}}$, the transverse Lyapunov exponent evaluated with respect to a trajectory in the chaotic attractor in the synchronization manifold $\mathscr{M}$ is nearly zero. Therefore, unstable periodic orbits in $\mathscr{M}$ represents a large fraction of all periodic orbits in the attractor in the full phase space.

${ }^{5}$ The severeness of unstable dimension variability can be quantified by using the following contrast measure [39]: $C_{p}=$ $\left|\frac{\mu_{2}(p)-\mu_{1}(p)}{\mu_{2}(p)+\mu_{1}(p)}\right|$, where $\mu_{1,2}(p) \equiv \sum_{j=1}^{N_{1,2}(p)} \mathrm{e}^{-\lambda_{1}^{(j)}(p) p}, \quad N_{1}(p)$ and $N_{2}(p)$ are the numbers of periodic orbits of period $p$ with one and two unstable directions, respectively. The quantities $\mu_{1,2}(p)$ are then the weighted numbers of period- $p$ orbits with one and two unstable directions, respectively. When there is no unstable dimension variability, we have either $N_{2}(p)=0$ or $N_{1}(p)=0$, which yields $C_{p}=1$. The contrast $C_{p}$ starts to decrease from one when unstable dimension variability occurs, and the worst case is $C_{p}=0$, corresponding to the situation where unstable dimension variability is most severe $\left[\mu_{1}(p)=\mu_{2}(p)\right]$. Numerical computations of contrasts for periodic orbits of different periods reveal that the values of these contrasts are near zero in parameter regimes where transitions to chaos with multiple positive Lyapunov exponents occur, indicating the presence of severe unstable dimension variability near the transitions.
}

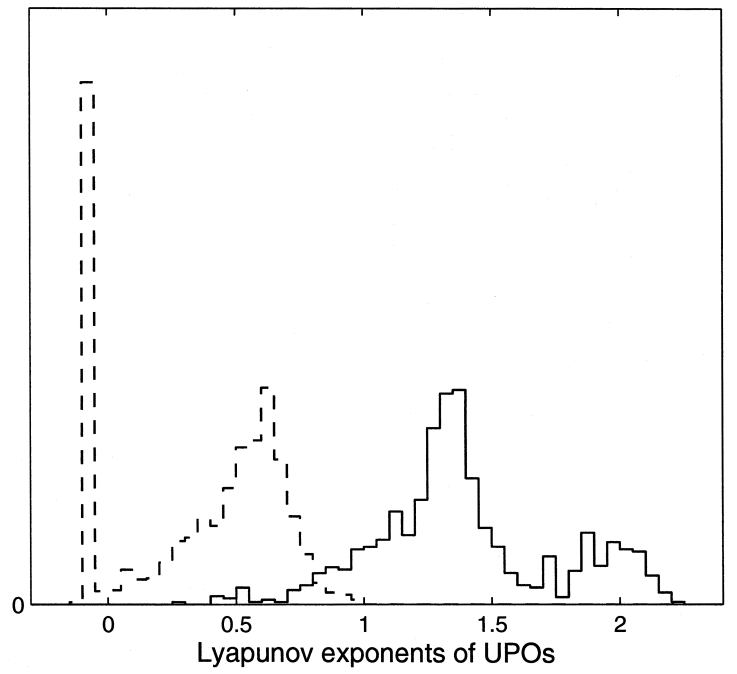

Fig. 3. Histograms of the first (solid line) and second (dashed line) largest Lyapunov exponents of all periodic orbits of period 7 in the full four-dimensional system Eq. (5).

punov exponents $\lambda_{1}^{j} e^{-\lambda_{1}^{j} p} \quad$ and $\lambda_{2}^{j} e^{-\lambda_{1}^{j} p} \quad(j=$ $1, \ldots, N_{7}$ ) for all periodic orbits of period 7 . We see that the second largest Lyapunov exponent of the periodic orbits can be either positive or negative, indicating unstable dimension variability.

In summary, we have presented a heuristic theory and solid numerical evidence for the characterization of the transition to chaos with multiple positive Lyapunov exponents by unstable periodic orbits. Our main conclusion is that this transition is typically accompanied by severe unstable dimension variability. This provides evidence, at the level of unstable periodic orbits, for the smooth behavior of Lyapunov exponents (except for the largest one) through zero in high-dimensional chaotic systems [7-12]. The transition is thus different from most transitions to low-dimensional chaos in that the former involves an infinite number of periodic orbits. We remark that an explicit verification of unstable dimension variability requires precise computation of a large number of unstable periodic orbits embedded in the chaotic attractor, which is difficult. We are able to test our argument due largely to our improved version [30] of the Schmelcher-Diakonos algorithm [28,29].

We stress that, intuitively, when a chaotic system evolves from having one positive Lyapunov expo- 
nent to having more than one such exponent, there has to be a variability in the unstable dimensions among unstable periodic orbits embedded in the chaotic set, and the continuous behavior in the Lyapunov exponents near this transition is a natural consequence of the unstable dimension variability. This paper provides evidence for this intuition. However, just as there are different routes to lowdimensional chaos, there can be distinct scenarios to high-dimensional chaos. The one through unstable dimension variability investigated in this paper is just one such scenario that is expected to occur commonly in chaotic systems.

\section{Acknowledgements}

This work was supported by AFOSR under Grant No. F49620-98-1-0400 and by NSF under Grant No. PHY-9722156.

\section{References}

[1] M.J. Feigenbaum, J. Stat. Phys. 19 (1978) 25.

[2] Y. Pomeau, P. Manneville, Commun. Math. Phys. 74 (1980) 189.

[3] C. Grebogi, E. Ott, J.A. Yorke, Physica D 7 (1983) 181.

[4] D. Ruelle, F. Takens, Commun. Math. Phys. 20 (1971) 167.

[5] D. Ruelle, F. Takens, Commun. Math. Phys. 23 (1971) 343.

[6] S. Newhouse, D. Ruelle, F. Takens, Commun. Math. Phys. 64 (1978) 35.

[7] A. Torcini, A. Politi, G.P. Puccioni, G. D’Alessandro, Physica D 53 (1991) 85

[8] T. Kapitaniak, W.-H. Steeb, Phys. Lett. A 152 (1991) 33.

[9] S. Lepri, G. Giacomelli, A. Politi, F.T. Arecchi, Physica D 70 (1993) 235.

[10] F. Cecconi, A. Politi, Phys. Rev. E 56 (1997) 4998.

[11] A. Politi, A. Witt, Phys. Rev. Lett. 82 (1999) 3034.
[12] M.A. Harrison, Y.-C. Lai, Phys. Rev. E 59 (1999) R3799.

[13] S.P. Dawson, Phys. Rev. Lett. 76 (1996) 4348.

[14] E. Barreto, B. Hunt, C. Grebogi, J.A. Yorke, Phys. Rev. Lett. 78 (1997) 4561.

[15] D. Auerbach, C. Grebogi, E. Ott, J.A. Yorke, Phys. Rev. Lett. 69 (1992) 3479.

[16] V. Petrov, M.J. Crowley, K. Showalter, Phys. Rev. Lett. 72 (1994) 2955.

[17] G.A. Johnson, M. Löcher, E. Hunt, Phys. Rev. E 51 (1995) R1625.

[18] M. Ding, W. Yang, V. In, W.L. Ditto, M.L. Spano, B. Gluckman, Phys. Rev. E 53 (1996) 4334.

[19] M.A. Rhode, J. Thomas, R.W. Rollins, A.J. Markworth, Phys. Rev. E 54 (1996) 4880.

[20] L. Kocarev, U. Parlitz, Phys. Rev. Lett. 77 (1996) 2206.

[21] Y.-C. Lai, Phys. Rev. E 55 (1997) R4861.

[22] L. Pecora, T.L. Carroll, G. Johnson, D. Mar, Phys. Rev. E 56 (1997) 5090.

[23] D. Auerbach, P. Cvitanović, J.-P. Eckmann, G.H. Gunaratne, I. Procaccia, Phys. Rev. Lett. 58 (1987) 2387.

[24] R. Abraham, S. Smale, Proc. Symp. Pure Math. 14 (1970) 5.

[25] S.P. Dawson, C. Grebogi, T. Sauer, J.A. Yorke, Phys. Rev. Lett. 73 (1994) 1927.

[26] T. Sauer, C. Grebogi, J.A. Yorke, Phys. Rev. Lett. 79 (1997) 59.

[27] E.J. Kostelich, I. Kan, C. Grebogi, E. Ott, J.A. Yorke, Physica D 109 (1997) 81.

[28] P. Schmelcher, F.K. Diakonos, Phys. Rev. Lett. 78 (1997) 4733.

[29] P. Schmelcher, F.K. Diakonos, Phys. Rev. E 57 (1998) 2739.

[30] R. Davidchack, Y.-C. Lai, Phys. Rev. E 60 (1999) 6172.

[31] Ja.B. Pesin, Sov. Math. Dokl. 17 (1976) 196.

[32] R.S. MacKay, J.D. Meiss (Eds.), Hamiltonian Dynamical Systems: A Reprint Selection, Adam Hilger, Bristol, 1987.

[33] D. Ruelle, Chaotic Evolution and Strange Attractors, Cambridge Univ. Press, New York, 1989.

[34] J.-P. Eckmann, D. Ruelle, Rev. Mod. Phys. 57 (1985) 617

[35] C. Grebogi, E. Ott, J.A. Yorke, Phys. Rev. A 37 (1988) 1711.

[36] Y.-C. Lai, Y. Nagai, C. Grebogi, Phys. Rev. Lett. 79 (1997) 652 .

[37] K. Ikeda, Opt. Commun. 30 (1979) 257.

[38] S.M. Hammel, C.K.R.T. Jones, J. Moloney, J. Opt. Soc. Am. B 2 (1985) 552.

[39] Y.-C. Lai, Phys. Rev. E 59 (1999) R3807. 